\title{
Decellularized Bone Extracellular Matrix and Human Dental Pulp Stem Cells as a Construct for Bone Regeneration
}

\author{
Francesco Paduano', Massimo Marrelli², Noura Alom ${ }^{3}$, Mahetab Amer ${ }^{3}$, Lisa J \\ White $^{3}$, Kevin M Shakesheff*3 \& Marco Tatullo*1
}

\author{
${ }^{1}$ Tecnologica Research Institute, Biomedical Section, Crotone, Italy \\ ${ }^{2}$ Unit of Maxillofacial Surgery, Calabrodental, Crotone, Italy \\ ${ }^{3}$ School of Pharmacy, University of Nottingham, Nottingham NG7 2RD, UK
}

*Correspondence should be addressed to:

\section{Kevin M. Shakesheff or Marco Tatullo}

kevin.Shakesheff@nottingham.ac.uk

Wolfson Centre for Stem Cells, Tissue Engineering, and Modelling (STEM) Centre for Biomolecular Sciences University of Nottingham University Park

Nottingham NG7 2RD, UK Tel: +44 (0) 1159515 104/121

marco.tatullo@tecnologicasrl.com

Research and Development in Biomedicine, Stem Cells Unit, TECNOLOGICA Research Institute, st. E. Fermi Loc. Passovecchio I. Z., 88900 Crotone (KR), Italy. Tel: 00393498742445 


\section{Abstract}

Dental pulp tissue represents a source of mesenchymal stem cells (MSCs) that have a strong differentiation potential towards the osteogenic lineage. The objective of the current study was to examine in vitro osteogenic induction of dental pulp stem cells (DPSCs) cultured on hydrogel scaffolds derived from decellularized bone extracellular matrix (bECM) compared to collagen type I (Col-I), the major component of bone matrix. DPSCs in combination with bECM hydrogels were cultured under three different conditions: basal medium, osteogenic medium and medium supplemented with growth factors (GFs) and cell growth, mineral deposition, gene and protein expression were investigated. The DPSCs/bECM hydrogel constructs cultured in basal medium showed that cells were viable after three weeks and that the expression of runt-related transcription factor $2(R U N X-2)$ and bone sialoprotein $(B S P)$ were significantly upregulated in the absence of extra osteogenic inducers compared to Col-I hydrogel scaffolds. In addition, the protein expression levels of BSP and osteocalcin (OCN) were higher on bECM with respect to Col-I hydrogel scaffolds. Furthermore, DPSCs/bECM hydrogels cultured with osteogenic or GFs supplemented medium displayed a higher upregulation of the osteo-specific markers compared to Col-I hydrogels in identical media. Collectively, our results demonstrate that bECM hydrogels might be considered as suitable scaffolds to support osteogenic differentiation of DPSCs.

\section{Keywords:}


Bone extracellular matrix (bECM); dental pulp stem cells (DPSCs); hydrogels; scaffolds; bone regeneration.

\section{Introduction}

Current treatment options to repair bone defects due to trauma, degenerative diseases, severe infections and tumours are based on autologous or allogeneic bone grafts.[1,2] Although autologous bone grafts remain the gold standard, they are limited by morbidity at the donor site and availability of graft material.[3,4] Allogeneic bone grafts may provide an alternative treatment, but the risk of potential immunogenic responses, disease transmission, reduced osteoinductive characteristics, availability limitations and ethical/religious issues limit their use.[3,4] Hence, there is the need for alternative materials to treat bone defects.[5,6].

Recent research has attempted to identify synthetic or natural materials which mimic the properties of the native bone microenvironment to act as bone graft substitutes.[7-9] Natural materials, including collagen and scaffolds composed of the extracellular matrix have recently been considered as cell delivery systems for bone repair.[10]

Cell-matrix interactions induce signalling essential for cell behaviour, making ECM composition a crucial factor in stem cell differentiation.[11,12] Proliferation and lineagespecific differentiation of mesenchymal stem cells (MSCs) are triggered by various factors including the ECM.[13] Thus, several studies have proposed the use of biological scaffolds composed of decellularized ECM to induce osteogenic differentiation of MSCs.[14,15]

ECM scaffolds consist of functional and structural proteins such as laminin and collagen, along with growth factors, proteoglycans, glycosaminoglycans and glycoproteins.[16] 
While there are several common ECM components, the detailed composition of the ECM varies for each tissue type.[17] ECM scaffolds derived from bone are principally composed of collagen type I, with smaller amounts of collagen III, collagen V and adhesion proteins such as fibronectin, vitronectin and proteoglycans such as decorin and hyaluronan.[18] Bone ECM also contains pro-inflammatory cytokines, growth factors of the TGF- $\beta$ family, several BMPs, FGFs, and angiogenic growth factors including VEGF.[7]

In this context, it was recently reported that tissue-specific hydrogels derived from decellularized and demineralized bovine bone extracellular matrix (bECM) possess distinct mechanical and biological properties, including osteogenic functionality and have the potential for clinical use in the near future.[19]

More recently, we showed that bECM hydrogel scaffolds were also able to stimulate odontogenic differentiation of human dental pulp stem cells (DPSCs) by upregulating the expression levels of the odonto/osteogenic-specific genes.[20] Additional studies with bECM hydrogel scaffolds have shown the efficacy of bone-derived ECM hydrogels with incorporated growth factors to augment bone formation within the ex vivo chick femoral defects.[3,4] Moreover, Gothard et al. showed that alginate/bECM hydrogel scaffolds in combination with adult human bone marrow stromal cells (HBMSCs) were able to provide in vivo tissue mineralisation and bone formation.[21]

Recently, DPSCs have been considered as a source of cells for bone engineering due to their availability and high capacity of in vitro expansion.[12,22,23] Moreover, DPSCs are able to differentiate into bone-like tissues when seeded on scaffolds in animal models [24,25] and are able to regenerate bone in human grafts.[26,27] Taken together, these 
studies prompted us to analyse whether a combination of DPSCs and bECM could be used as bone graft substitute for the treatment of bone defects. Therefore, the objective of the current study was to examine the osteogenic capacity of the decellularized bECM hydrogel scaffolds derived from bovine bone using DPSCs. To that end, DPSCs/bECM hydrogel constructs have been compared to Col-I, the most commonly purified ECM component. Col-I hydrogels at the same concentrations were used as a comparator for three reasons. Firstly, it is the most abundant ECM protein in many adult tissues, including bone, and is easily extracted using enzymatic or acidic methods.[28] Secondly, collagen-based scaffolds are among the most extensively used scaffolds in the clinical application with different forms including native, cross-linked and gel forms.[29] Thirdly, purified Col-I derived scaffolds are FDA approved for several clinical applications.[30] Growth factors such as FGFb [31,32] and EGF [32,33] have been used to promote osteogenic differentiation of DPSCs. We hypothesised that the combination of bECM hydrogels with growth factors or osteogenic supplements could more effectively induce the osteogenic differentiation of human DPSCs than bECM hydrogels alone. Thus, the first aim of this study was to evaluate if bECM hydrogels are sufficient to induce osteogenic differentiation of DPSCs without the addition of osteogenic supplements. The second was to determine if osteogenic supplements or growth factors were able to enhance osteogenic differentiation of DPSCs/bECM hydrogel constructs. To these ends, we analysed the effects of bECM hydrogel scaffolds upon osteogenic differentiation of DPSCs in the presence or absence of extra osteogenic inducers (growth factors or osteogenic medium), by observing changes in osteo-specific genes and protein expression. 


\section{Materials and Methods}

\subsection{Hydrogels preparation}

Decellularized and demineralized bECM was prepared from bovine bone as described previously.[3,4,19-21] Briefly, liquid nitrogen was used to freeze and fragment bovine cancellous bone. Then, cancellous fragments were subsequently demineralized using a solution of $0.5 \mathrm{M} \mathrm{HCl}$ at $\mathrm{RT}$ for $24 \mathrm{~h}$. After demineralization, a solution of chloroform/methanol was used to remove lipids. Then, demineralized powder was decellularized by $24 \mathrm{~h}$ of agitation in $0.05 \%$ Trypsin $/ 0.02 \%$ EDTA at $37^{\circ} \mathrm{C}, 5 \% \mathrm{CO}_{2}$. To obtain bECM digests, powdered demineralized and decellularized bone was combined with $1 \mathrm{mg} / \mathrm{mL}$ pepsin in $0.01 \mathrm{M} \mathrm{HCl}$ for a final concentration of $10 \mathrm{mg} / \mathrm{mL}$. Then, the suspension was stirred at RT for $96 \mathrm{~h}$.

Gelation of bECM was achieved by neutralizing the $\mathrm{pH}$ and salt concentration of the pepsin digests at $4{ }^{\circ} \mathrm{C}$ followed by incubation at $37^{\circ} \mathrm{C}$ for $1 \mathrm{~h}$ (Supplemental material, Figure S1) as previously described.[19] Neutralization of the required digest volume occurred by addition of one tenth of the digest volume of $\mathrm{NaOH}(0.1 \mathrm{~N})$ and one-ninth of the digest volume of PBS (10X). To determine whether different concentrations of bECM could influence the osteogenic differentiation of DPSCs, we prepared different concentrations of bECM. In addition, due to the fact that type I collagen is currently considered the gold standard in the field of tissue engineering,[34] we prepared hydrogel scaffolds at the same concentrations of Col-I (Supplemental material, Figure S2). Concentrations of $3,4,6$ and $8 \mathrm{mg} / \mathrm{mL}$ bECM were prepared at $4{ }^{\circ} \mathrm{C}$ and transferred to plates. Col-I hydrogels used in this work were made following manufacturer's instructions (Corning). 


\subsection{Rheological characterization of bECM hydrogels}

The rheological characteristics of bECM and collagen type I hydrogels were determined using a Physica MCR 301 rheometer (Anton Paar, Hertford, UK). Pre-gel solutions at $4^{\circ} \mathrm{C}$ were placed between $50 \mathrm{~mm}$ parallel plates separated by a $0.2 \mathrm{~mm}$ measuring gap. The plates were pre-cooled within a humidified chamber to $4^{\circ} \mathrm{C}$ and were then warmed to $37^{\circ} \mathrm{C}$ during the first 75 seconds of each measurement run. A 20 -minute time course experiment was performed, during which the samples were subjected to an oscillatory strain of $1 \%$ at a constant angular frequency of $1 \mathrm{rad} \mathrm{s}^{-1}$ with readings taken every 30 seconds.

\subsection{Dental pulp stem cell cultures}

The protocol for the isolation of dental pulp stem cells (DPSCs) was approved by the Ethical Committee of Calabrodental Dental Clinic, Crotone, Italy. All subjects enrolled in this research have responded to an Informed Consent which has been approved by the Ethical Committee of Calabrodental Dental Clinic (ethical agreement number CBD008/TRI/2015). Human third molars were extracted from healthy donors (age 20-25).

DPSCs were isolated as previously described.[20] Briefly, teeth were immersed and washed twice using antibiotics (100 U/mL penicillin and $100 \mu \mathrm{g} / \mathrm{mL}$ streptomycin). The dental pulp tissue was separated from crown and root using an excavator. Then, the tissue was washed twice with sterile PBS and dissected with a scalpel into small pieces. Subsequently, to generate single cells suspension, pulp fragments were dissociated enzymatically with type I collagenase $(3 \mathrm{mg} / \mathrm{mL})$ and dispase $(4 \mathrm{mg} / \mathrm{mL})$ for 60 minutes at $37^{\circ} \mathrm{C}$. Then, the cell suspension was filtered through $70 \mu \mathrm{M}$ Falcon strainers (Becton Dickinson). After filtration, cells were pelleted, seeded on culture dishes, and incubated in alpha-MEM culture medium supplemented with $10 \%$ foetal bovine serum (FBS; 
Invitrogen), $2 \mathrm{mM}$ glutamine (Gibco), $100 \mathrm{U} / \mathrm{mL}$ penicillin, and $100 \mathrm{mg} / \mathrm{mL}$ streptomycin (Invitrogen). Cells were incubated at $37^{\circ} \mathrm{C}$ and $5 \% \mathrm{CO}_{2}$ and the medium was changed every $48 h$.

In this study, we used CFU-F derived cells capable of differentiating in at least three mesenchymal lineages in vitro. To generate CFU-F enriched cultures, cells were seeded at low density and cultured until colonies appeared. All the colonies were then trypsinized and further expanded in culture. Cells from passage 2-4 were employed in the study. The multi-differentiation capacity of these stem cells in different inductive media was demonstrated in our previous studies.[35,36]

\subsection{DPSC seeded hydrogels}

Once the hydrogels were formed, $0.5 \times 10^{5}$ DPSCs in a $1 \mathrm{ml}$ suspension were added to the surface of the gels (Supplemental material, Figure S3). Twenty-four hours after seeding, the culture medium was replaced with basal medium, osteogenic medium or medium supplemented with growth factors (GFs) every 24 hours in the first two days of preparation and then every second day after that. Subsequently, the DPSCs/bECM or DPSCs/Col-I hydrogel constructs were cultured for 3 weeks. The basal medium was composed of $\alpha$-MEM supplemented with $10 \%$ foetal bovine serum (FBS), $2 \mathrm{mM}$ glutamine, $100 \mathrm{U} / \mathrm{mL}$ penicillin, and $100 \mathrm{mg} / \mathrm{mL}$ streptomycin as described above for DPSCs isolation.[20] Osteogenic medium was composed of $\alpha$-MEM, 20\% FBS, $0.2 \mathrm{mM}$ L-ascorbic acid-2-phosphate (Sigma), $100 \mathrm{nM}$ dexamethasone (Sigma), $10 \mathrm{mM} \beta$ glycerophosphate (Sigma), $100 \mathrm{U} / \mathrm{mL}$ penicillin, $0.1 \mathrm{mg} / \mathrm{mL}$ streptomycin, and 0.25 $\mathrm{mg} / \mathrm{mL}$ amphotericin B. The growth factor-supplemented medium was composed of $\alpha$ MEM, 10\% FBS, 20 ng/mL Epidermal Growth Factor (EGF, Invitrogen) and $40 \mathrm{ng} / \mathrm{mL}$ 
Fibroblast Growth Factor-basic (FGFb, Invitrogen). DPSCs were seeded on Col-I hydrogels with the same protocol used for the bECM hydrogels.

\subsection{Live/Dead Staining}

DPSCs/hydrogel constructs 3, 7, 14 and 21 days post seeding $(n=24)$ were incubated with live/dead staining solution that was composed of fluorescein diacetate $(1 \mu \mathrm{g} / \mathrm{mL})$ and propidium iodide $(20 \mu \mathrm{g} / \mathrm{mL})$ at RT for 20 minutes. Then, cells were monitored using a Leica TCS/SP2 confocal microscope system. Cell viability has been measured by Accuchip cell counter kit using the PI standard method of dead-cell staining combined with advanced image analysis (Adam, Nanoentek). Briefly, bECM or Col-I hydrogels containing cells $(n=24)$ were rinsed three times with PBS and digested with $1 \mathrm{ml}$ of a solution containing $3 \mathrm{mg} / \mathrm{mL}$ type I collagenase (Gibco) and $4 \mathrm{mg} / \mathrm{mL}$ dispase (Gibco) in HBSS for 60 minutes at $37^{\circ} \mathrm{C}$ with regular agitation. Then, recovered DPSCs were centrifuged and analysed by Accuchip cell counter kit.

\subsection{Flow cytometry analysis}

DPSCs cultured on bECM were analysed for the expression of MSC-related cell surface markers as previously described.[20,36,37] Briefly, 5X10 5 DPSCs were stained with FITC/PE/APC-conjugated primary antibodies on ice for 30 minutes prior to being analysed on a NAVIOS instrument (Beckman Coulter). The antibodies used were PE mouse anti-human CD13, APC mouse anti-human CD29, FITC mouse anti-human CD44, FITC mouse anti-human CD73, PE mouse anti-human CD90, APC mouse anti-human CD105, PE mouse anti-human CD146, APC-H7 mouse anti-human CD45 and PE mouse anti-human HLA-DR. All fluorochrome-conjugated antibodies used were purchased from Becton Dickinson (BD). Mouse IgG1-FITC, IgG1-PE, IgG1-APC and IgG1-APC-H7 were used as isotype controls. 
For intracellular staining of osteopontin (OPN), osteocalcin (OSC) and bone sialoprotein (BSP),[38] bECM or Col-I Hydrogels containing cells were washed three times in PBS, and digested with $1 \mathrm{ml}$ of solution containing $3 \mathrm{mg} / \mathrm{mL}$ type I collagenase (Gibco) and 4 $\mathrm{mg} / \mathrm{mL}$ dispase (Sigma) in Hank's Balanced Salt Solution (HBSS) for $1 \mathrm{~h}$ at $37^{\circ} \mathrm{C}$ with regular agitation. Recovered cells were centrifuged, washed and fixed with $4 \%$ (w/v) paraformaldehyde (Sigma) for 20 minutes at $4{ }^{\circ} \mathrm{C}$ and then permeabilized with a solution containing $0.1 \%$ Triton X-100 (Sigma) in PBS for 5 minutes. After blocking with 3\% bovine serum albumin (BSA; Sigma) for $30 \mathrm{~min}$ at RT, the cells were washed and incubated with indirect or direct fluorescent-conjugated antibodies. The primary antibodies used were: Phycoerythrin (PE)-conjugated anti-OPN and FITC-conjugated anti-OSC (R\&D Systems), and rabbit anti-BSP (Abcam). The secondary antibody was anti-rabbit FITC purchased from Abcam. For negative controls, cells were stained with an isotype control antibody. Cells were labelled with fluorescence-conjugated antibodies against OPN, OCN or with isotype controls. For BSP, cells were labelled with an antibody against BSP or with isotype control, followed by detection with Alexa 488-conjugated secondary antibody. Labelled cells were analysed by flow cytometry using a NAVIOS instrument (Beckman Coulter) and all data analysed using FlowJo vX0.7 software. Histograms show the geometric mean fluorescence intensity (GMFI). The total number of samples used in this assay is shown in Table S1 (supplemental material). $n=8$ constructs were used to obtain the results in section $3.3, n=4$ were used to obtain the results in section 3.4 and $n=4$ were used to obtain the results in section 3.5. The number of controls (TCPS) used in each results section are indicated in Table S1. 


\subsection{RNA isolation and quantitative real-time PCR (qRT-PCR)}

RNA isolation and qRT-PCR were performed as previously described.[20,39] See supplemental material for further details. The specific primers used for the amplification of runt-related transcription factor $2(R U N X-2)$, bone sialoprotein $(B S P)$, osteopontin $(O P N)$, osteocalcin $(O C N)$ and HPRT are shown in Table 1. $\mathrm{n}=24$ constructs were used to obtain the results in section $3.3, \mathrm{n}=12$ were used to obtain the results in section 3.4 and $\mathrm{n}=12$ were used to obtain the results in section 3.5. The number of controls (TCPS) used in each results section are indicated in Table S1.

\subsection{Immunofluorescence analysis}

DPSCs were cultured on bECM hydrogels $(3,4,6,8 \mathrm{mg} / \mathrm{mL})$ and Col-I hydrogels $(3,4$, $6,8 \mathrm{mg} / \mathrm{mL}$ ) for 3 weeks ( $\mathrm{n}=24$, Table S1). Subsequently, DPSCs/bECM hydrogel constructs were rinsed with PBS and fixed in $4 \%$ paraformaldehyde for 20 min at RT as described previously.[36] After washing three times with PBS, the cells were permeabilized with $0.2 \%$ Triton X-100 (Sigma) in PBS for 10 min at RT. The cells were then washed three times with PBS and blocked with 3\% BSA for $30 \mathrm{~min}$ at RT. The DPSCs/bECM hydrogel constructs were subsequently incubated for $12 \mathrm{~h}$ with indirect or direct fluorescent-conjugated antibodies at $4{ }^{\circ} \mathrm{C}$. The primary antibodies used were FITCconjugated anti-OCN (R\&D Systems), and rabbit anti-BSP (Abcam). The secondary antibody was anti-rabbit FITC purchased from Abcam. For negative controls, cells were stained with an isotype control antibody. The cell nuclei were stained with a $1 \mu \mathrm{g} / \mathrm{mL}$ solution of 4,6-diamidino-2-phenylindole (DAPI; Sigma) for $5 \mathrm{~min}$. The cells were subsequently mounted with anti-fading medium (ProLong Antifade; Invitrogen) and 
observed by confocal microscopy (Leica; TCS SP5). The samples were not sectioned and all images ( $\mathrm{n}>5$ fields for sample) were displayed as maximum intensity $\mathrm{z}$-projections.

\subsection{Von Kossa Staining}

DPSCs/bECM hydrogel constructs $(n=24)$ cultured for 3 weeks were washed two times with phosphate-buffered saline and fixed in $10 \%$ formalin prior to incubation with silver nitrate. Subsequently, Von Kossa staining kit was used to visualise the formation of the mineralized matrix (Bio-Optica).

\subsection{Alizarin Red}

DPSCs/bECM hydrogel constructs $(n=24)$ cultured for 3 weeks were washed once with PBS and fixed with 4\% paraformaldehyde (Sigma) for 15 min at RT. After washing thrice with PBS, an aqueous solution of $5 \mathrm{mg} / \mathrm{mL}$ Alizarin red $\mathrm{S}$ (Sigma) was added to the DPSCs/hydrogel constructs for 30 minutes. Then, DPSCs/hydrogel constructs were washed twice with $\mathrm{H}_{2} \mathrm{O}$ for 5 minutes and were analysed by microscopy.

\section{11. Statistical analysis}

A total of 208 DPSCs/hydrogel constructs were analysed in this study (Supplemental material, Table S1). Results were presented as means \pm standard deviations from three independent experiments. The significance of differences between groups was evaluated by one-way analysis of variance (ANOVA) followed by Dunnett's test for multiple comparisons. Analyses were conducted using the GraphPad Prism software package (Graphpad Software Inc). Values were considered statistically significant if $P<0.05$. 


\section{Results}

\subsection{Rheology of bECM hydrogels}

Hydrogels were prepared from bECM at concentrations ranging from 3-8 mg/mL (Figure 1A). The rheological characteristics of the bECM hydrogels were determined using a parallel plate rheometer and compared to collagen type I hydrogels of the same concentrations $(4,6$ and $8 \mathrm{mg} / \mathrm{mL})$. In each case, the storage $\left(\mathrm{G}^{\prime}\right)$ and loss $\left(\mathrm{G}^{\prime \prime}\right)$ modulus of the hydrogels increased after the pepsin digests (or pre-gel collagen type I solutions) were neutralized and the temperature was increased from $4{ }^{\circ} \mathrm{C}$ to $37^{\circ} \mathrm{C}$. Solid-like behaviour was confirmed since the storage modulus was greater than the loss modulus by a factor of approximately 10 for the bECM hydrogels and a factor of approximately 20 for the collagen type I hydrogels (Figures 1B, C and D). The bECM and Col-I hydrogels showed an increase in rate of gelation with increasing concentration wherein bECM and Col-I had storage moduli of $59.3 \pm 17.5 \mathrm{~Pa}$ and $58.5 \pm 5.0 \mathrm{~Pa}$, respectively, at $4 \mathrm{mg} / \mathrm{mL}$ and $145.1 \pm 65.7 \mathrm{~Pa}$ and $454.7 \pm 101.3 \mathrm{~Pa}$ at $8 \mathrm{mg} / \mathrm{mL}$.

\section{2. bECM supports cell attachment and growth of DPSCS}

Human mesenchymal stem cells derived from dental pulp (DPSCs) were first characterised using flow cytometry to confirm their mesenchymal phenotype, prior to seeding on bECM hydrogels. FACS analysis showed positive expression of cell surface markers of MSCs including CD13, CD29, CD44, CD73, CD90, CD105 and CD146. Negative expression was demonstrated for CD45 and HLA-DR (Figure 2A). DPSCs were seeded onto bECM hydrogels $(3,4,6,8 \mathrm{mg} / \mathrm{mL})$, and the distribution of cells was compared to those cultured on Col-I hydrogels or tissue culture polystyrene (TCPS). DPSCs spontaneously formed aggregates when cultured on $3,4,6,8 \mathrm{mg} / \mathrm{mL}$ bECM or 
Col-I hydrogels compared to TCPS (Figure 2B and supplemental material, Figures S3 and S4). DPSCs cultured on bECM hydrogels were viable after 21 days as shown by live/dead staining (Figure 2C). There were only a few DPSCs that were positive for Ethidium homodimer III (supplemental material, Figure S5), signifying low numbers of dead cells. Moreover, by day 7 of culture, the percentages of living cells in 3, 4, 6 and 8 $\mathrm{mg} / \mathrm{mL}$ bECM hydrogels were 95, 94, 94 and 92\%, respectively, with no statistical difference compared to Col-I hydrogels or TCPS (3 mg/mL Col-I, 96\%; 4 mg/mL Col-I, 96\%; 6 mg/mL Col-I, 94\%; 8 mg/mL Col-I, 92\%; TCPS, 94\%, Figure 2D).

\section{3. bECM hydrogels upregulate early, intermediate and late osteogenic markers of DPSCs in basal culture conditions}

To evaluate if the bECM hydrogels influenced the osteodifferentiation of DPSCs, cells were cultured in basal medium on the surface of $3,4,6,8 \mathrm{mg} / \mathrm{mL}$ bECM and Col-I hydrogels for 3 weeks. DPSCs seeded on the flask surface (TCPS) with basal medium or osteogenic medium were used as controls. The mRNA expression levels of Runt-related transcription factor $2(R U N X-2)$, bone sialoprotein $(B S P)$, osteopontin $(O P N)$ and osteocalcin $(O C N)$, four markers specific for osteogenic differentiation, were upregulated in both bECM (up to 2-, 2.5-, 10- and 8.6-fold, respectively) and Col-I hydrogel cultures (up to 1.3-, 1.4-, 9.2- and 6.1-fold, respectively) compared to TCPS cultured in basal medium, indicating the suitability of the both hydrogel scaffolds for osteogenic differentiation of DPSCs (Figure 3A). However, the expression levels of RUNX-2 and $B S P$ were significantly greater on the $4 \mathrm{mg} / \mathrm{mL}$ bECM hydrogel compared with Col-I at the same concentration. In addition, the expression of mRNA for $R U N X-2$ and $B S P$ were higher on the $4 \mathrm{mg} / \mathrm{mL}$ bECM hydrogel (2- and 2.6-fold, respectively) compared to DPSCs seeded on TCPS and cultured in osteogenic medium (Figure 3A). Results 
demonstrated that $O C N$ was strongly upregulated in cells seeded on bECM (up to 8.6fold) and Col-I hydrogels (up to 6.1-fold) compared to TCPS cultured in the basal medium. Conversely, DPSCs seeded on TCPS and cultured in osteogenic medium expressed higher levels of $O C N$ (5.2-fold) compared to both DPSCs/hydrogel constructs (Figure 3A). Protein expression analysis confirmed real-time reverse transcription-PCR (qRT-PCR) studies: flow cytometry revealed upregulation of OPN, OCN and BSP in DPSCs cultured on both bECM (up to 3.8-, 2.6- and 2-fold, respectively) and Col-I hydrogel cultures (up to 4.4-, 1.9- and 1.2-fold, respectively) compared to TCPS (Figure 3B). Moreover, the protein expression levels of BSP were greater on the $4 \mathrm{mg} / \mathrm{mL} \mathrm{bECM}$ hydrogel (3-fold) compared with Col-I at the same concentration and the expression of protein for OPN and BSP were higher on the $4 \mathrm{mg} / \mathrm{mL}$ bECM hydrogel (1.5- and 1.4fold, respectively) compared to DPSCs seeded on TCPS and cultured in osteogenic medium. As previously observed for $O C N$ gene expression, DPSCs seeded on TCPS and cultured in osteogenic medium expressed higher levels of OCN protein (up to 2-fold) compared to both DPSCs/hydrogel constructs (Figure 3B).

In addition, immunofluorescence analysis showed the higher protein expression level of BSP and OCN in DPSCs cultured on bECM compared to Col-I hydrogels (Figure 3C). Moreover, the ability of both DPSCs/hydrogel constructs to form mineralized matrix was analysed by using Von Kossa and Alizarin Red staining. Results demonstrated that both types of DPSCs/bECM hydrogel constructs were able to form mineral deposits after 3 weeks of culture (Figures 4A, B). Taken together, the results of the present study demonstrated that bECM hydrogels combined with DPSCs are sufficient to induce osteogenic differentiation without the addition of osteogenic supplements. 


\subsection{Osteogenic supplements enhance osteogenic differentiation of DPSCs/bECM hydrogel constructs.}

In order to further assess the effects of the bECM hydrogels on the osteogenic differentiation of dental pulp stem cells, we analysed the osteogenic-specific genes expression of DPSCs cultured on $4 \mathrm{mg} / \mathrm{mL}$ bECM hydrogel after 3 weeks of differentiation in osteogenic medium. We decided to use bECM hydrogel at a concentration of $4 \mathrm{mg} / \mathrm{mL}$ since the gene expression levels of $R U N X-2$ and $B S P$ were increased at concentration. DPSCs grown in osteogenic medium on a flask surface (TPCS), and on $4 \mathrm{mg} / \mathrm{mL}$ bECM or Col-I hydrogels cultured in basal medium have been used as control conditions. Quantitative RT-PCR analyses (Figure 5A) revealed that the expression of $R U N X-2, B S P, O P N$ and $O C N$ were significantly higher in DPSCs seeded on bECM hydrogels cultured on osteogenic medium (2.2-, 3.6-, 1.6- and 3.6-fold, respectively) than in basal medium (Figure 5A). In DPSCs/bECM hydrogel constructs, the expression levels of $R U N X-2, B S P$ and $O P N$ were also considerably higher than cells cultured on TCPS with osteogenic medium.

The expression level of $O C N$ was increased in DPSCs/bECM scaffold constructs cultured in the osteogenic medium (3.6-fold) compared to those cultured on basal medium. However, the expression of $O C N$ in either bECM or Col-I hydrogels was lower (0.56and 0.27 -fold, respectively) than cells cultured on TCPS with osteogenic medium (Figure 5A). Importantly, DPSCs cultured on $4 \mathrm{mg} / \mathrm{mL}$ bECM hydrogel scaffold with osteogenic medium stimulated higher upregulation of the osteo-specific genes $(O C N, 2.1$-fold; $O P N$, 1.5 -fold; RUNX-2, 1.5 -fold) than on $4 \mathrm{mg} / \mathrm{mL}$ Col-I hydrogel cultured in identical medium.

To confirm these results, we measured the expression levels of osteogenic proteins in DPSCs seeded on $4 \mathrm{mg} / \mathrm{mL}$ bECM hydrogel cultured in basal medium or osteogenic 
medium compared to $4 \mathrm{mg} / \mathrm{mL}$ Col-I hydrogel or TCPS by flow cytometry.[38] Analysis revealed that the protein expression levels of BSP, OPN and OCN were higher in DPSCs seeded on $4 \mathrm{mg} / \mathrm{mL}$ bECM hydrogel cultured in the osteogenic medium (3.6-, 1.6- and 3.7-fold, respectively) than those in basal medium (Figure 5B). Importantly, results show that both BSP and OPN protein expression were upregulated in DPSCs grown on 4 $\mathrm{mg} / \mathrm{mL}$ bECM (2.62- and 2.4-fold) compared to TCPS-cultured cells treated with osteogenic medium. Moreover, the protein expression levels of BSP were greater on the $4 \mathrm{mg} / \mathrm{mL}$ bECM hydrogel cultured in osteogenic medium (3-fold) compared with Col-I in the same culture conditions.

\subsection{Growth factors affect the osteogenic differentiation of DPSCs in combination with bECM hydrogels by upregulating RUNX-2, BSP and OCN.}

To assess the influence of growth factors (GFs) on the osteogenic potential of DPSCs/hydrogel constructs, we evaluated the expression of osteo-specific genes in DPSCs cultured on $4 \mathrm{mg} / \mathrm{mL}$ bECM hydrogels with or without growth factors compared to controls. $R U N X-2$ and $B S P$ mRNA levels of DPSCs cultured on $4 \mathrm{mg} / \mathrm{mL} \mathrm{bECM}$ hydrogel scaffolds supplemented with growth factors increased significantly (2.3- and 35-fold, respectively) more than those cultured on TCPS in the same culture conditions, whereas a significant reduction in the mRNA expression of $O P N$ was detected in DPSCs cultured on bECM hydrogels or TCPS with GFs (Figure 6A). Compared to TCPS in the presence of GFs, the cells/bECM hydrogel constructs exhibited a significant increase in the expression of $O C N$ (2.41-fold). Similar results were obtained for protein expression of OPN, OCN and BSP in DPSCs cultured on $4 \mathrm{mg} / \mathrm{mL}$ bECM hydrogels (Figure 6B) in the presence of GFs with respect to controls (1.9-, 1.44-, and 2.4-fold increase compared 
to Col-I hydrogels and 5.3-, 5.4- and 1.9-fold increase compared to TCPS cultured in medium containing GFs, respectively). These results showed that the osteogenic differentiation of DPSCs cultured on bECM hydrogels is maintained also using a medium containing growth factors.

\section{Discussion}

To overcome the limitations of the existing methods to produce bone graft substitutes, $[2,40]$ new strategies include the culture of osteogenic cells on several types of scaffolds.[41,42] In this context, dental pulp stem cells (DPSCs) have been shown to differentiate into bone-like tissues in animal models when seeded on scaffolds.[43,44]

Gronthos et al. first isolated and characterised these stem cells [22] from impacted third molars demonstrating similarities to human bone marrow stromal cells (BMSCs) and suggested their application in musculoskeletal regenerative medicine. Recent studies demonstrated that the osteogenic potential of DPSCs was significantly greater than that of BMSCs and adipose tissue-derived stem cells (ADSCs).[45,46]

DPSCs are a potential source for bone tissue engineering and osteogenic capacity of these cells has been well demonstrated in vitro and in vivo. $[23,26]$ In fact, different studies have shown that DPSCs can differentiate under specific conditions into osteoblasts in vitro, and when implanted in immunocompromised animals can restore critical bone defects and produce bone.[47,48] Moreover, Papaccio's group have demonstrated that when DPSCs were transplanted into immunocompromised rats, the cells generated tissue structures similar to that observed in adult human bone including an adequate blood supply.[43] The osteogenic capacity of DPSCs has been utilised in combination with different scaffolds to produce bone-like tissues in several promising studies that further 
indicate the potential of DPSCs for bone regeneration and repair.[49]

Although DPSCs are available in limited amounts when compared with BMSCs or ADSCs, the process of stem cell isolation does not require to sacrifice a healthy tooth. In fact, third molars represent the most common source of DPSCs, since these teeth are often extracted for orthodontic reasons and are considered to be medical waste.[49,50] Moreover, DPSCs can be long-term cryopreserved and recovered; this renders them a potentially suitable and reliable source of cells. [51]

In the current study, we determined the effects of extracellular matrix (ECM) hydrogel scaffolds derived from bovine bone (bECM) [19] upon DPSCs cultured in basal conditions and in the presence of osteogenic medium or growth factors.

The results of the present study demonstrate that bECM hydrogels at different concentrations supported cell attachment and growth of DPSCs. Moreover, bECM scaffolds enhance osteogenic differentiation of human DPSCs by inducing an upregulation of osteo-specific genes $R U N X-2, B S P, O P N$ and $O C N$ and the corresponding proteins. These bECM hydrogels also improve the mineralized matrix formation of DPSCs probably by enhancing the expression of bone glycoproteins such as OPN and BSP, both involved in the formation of the mineralized matrix.[52] Importantly, the combination of bECM hydrogels with DPSCs is sufficient to induce osteogenic differentiation of DPSCs, without requiring additional osteogenic factors. We hypothesise that the mechanical properties of bECM, as well as the constitutive molecules and the biological activity of degradation products,[19] could be responsible for the osteogenic differentiation of DPSCs. Similar results have been shown in another work, in which an increase in mRNA expression of osteoblast-related genes was observed in 
DPSCs seeded on biocoral scaffolds using medium without the addition of other substances.[41]

Here, we observed that both BSP and OPN expression were higher in DPSCs/bECM hydrogel constructs cultured in basal medium compared to TCPS-cultured cells treated with osteogenic medium. However, at the same time, we observed that DPSCs/bECM hydrogel constructs expressed lower levels of OCN compared to TCPS cultured in osteogenic medium. We postulate that the early and intermediate osteogenic markers (RUNX-2, BSP, OPN),[53,54] but not the late osteogenic markers, such as OCN,[55] can be efficiently upregulated by bECM. Importantly, we observed also that the expression of the osteogenic markers RUNX-2 and BSP were higher in bECM than Col-I hydrogel constructs. These results indicate that bECM may be better suited for supporting osteogenic differentiation of DPSCs than Col-I hydrogels. We hypothesise that the differences observed between bECM and Col-I might be due to the different mechanical and biological properties of these scaffolds.[19] In the second part of this study, using a combination of GFs containing FGFb and EGF, we have observed that DPSCs seeded on bECM have a higher expression of osteogenic markers than those seeded on TCPS or Col-I. The results indicate that growth factors are capable of improving the osteogenic potential of bECM hydrogels. Collectively, our data show that bECM hydrogel scaffolds provide an enhanced environment for the osteogenic differentiation of DPSCs.

Our previous study demonstrated the potential of the bECM hydrogel scaffolds to upregulate the expression of odontogenic specific genes in DPSCs.[20] Here, we found that hydrogel scaffolds can also direct the osteogenic differentiation of DPSCs by upregulating the expression of the osteogenic markers at both mRNA and protein levels. 
Although the results support the potential use of our proposed cell-seeded hydrogel scaffolds in bone tissue engineering, there is no a positive dose-response relationship between protein content in the hydrogels $(3,4,6,8 \mathrm{mg} / \mathrm{mL})$ and osteogenic differentiation of the cells. We hypothesise that 6 and $8 \mathrm{mg} / \mathrm{mL}$ hydrogels contain a high concentration of bone-formation inhibitory factors such as sclerostin [56] or others bovine peptides that could hamper the osteogenic differentiation of DPSCs.

The present work is a preliminary study; further investigations are needed to demonstrate the biocompatibility and osteoconductivity of the DPSCs/bECM hydrogel constructs in vivo.

\section{Conclusions}

In this study, we aimed to measure the effect of bECM on osteogenic differentiation of DPSCs. Our results demonstrate that bECM hydrogels facilitated a significant upregulation of markers involved in osteogenesis, indicating that DPSCs are directed towards the osteogenic lineage. The ability of the bECM hydrogel to induce osteogenic differentiation of DPSCs without the addition of growth factors makes it an interesting biomaterial for bone tissue engineering applications.

Overall, the findings from our study have shown some encouraging results which provide proof-of-principle data suggesting that DPSCs/bECM hydrogel constructs can be used for bone regenerative purposes. However, further studies are needed to assess the clinical relevance of the DPSCs/bECM hydrogel constructs.

In conclusion, the DPSCs/bECM hydrogel constructs developed in this study could be considered promising candidates for the treatment of bone defects. 


\section{Supplemental material}

Supplemental Materials and Methods: RNA isolation and quantitative real-time PCR (qRT-PCR).

Table S1: Experimental design; treatment conditions, assignment of DPSCs/hydrogel constructs and response measures.

Figure S1. Representative images of bECM hydrogels used in this study.

Figure S2. Macroscopic appearance of Col-I hydrogels. Scale bar: $1 \mathrm{~cm}$.

Figure S3. Distribution of DPSCs cultured on bECM (4 and $8 \mathrm{mg} / \mathrm{mL}$ ). Phase contrast, scale bar: $100 \mu \mathrm{m}$.

Figure S4. Distribution of DPSCs cultured on tissue culture polystyrene (TCPS) at 1, 3, 7 and 14 days. Scale bar: $200 \mu \mathrm{m}$.

Figure S5. Live/dead analysis of cells/scaffold constructs 14 days after cell seeding in basal medium, GFs supplemented medium and osteogenic medium. Viable cells stained green and dead cells stained red after Calcein/AM-EthD-III staining. Cells were observed under fluorescence confocal microscope, scale bar: $100 \mu \mathrm{m}$.

\section{Acknowledgements}

This work was supported by the "ICARE Project - Infrastruttura Calabrese per la medicina Rigenerativa: generazione di biobanche per la criopreservazione di cellule staminali umane e di tessuto osseo per uso clinico e design e sviluppo di bioscaffold innovativi" under Grant number PON03PE_00009_2.2. LJW was funded by a Marie Curie International Outgoing Fellowship under REA grant agreement no. 624841. 


\section{Conflict of Interests}

The authors declare that they have no conflicts of interest. Professor Shakesheff is a consultant of Calabrodental SRL.

\section{References}

[1] Bauer TW, Muschler GF. Bone graft materials. An overview of the basic science. Clinical orthopaedics and related research. 2000:10-27.

[2] De Long WG, Jr., Einhorn TA, Koval K, et al. Bone grafts and bone graft substitutes in orthopaedic trauma surgery. A critical analysis. The Journal of bone and joint surgery American volume. 2007;89:649-58.

[3] Smith EL, Kanczler JM, Gothard D, et al. Evaluation of skeletal tissue repair, part 1: assessment of novel growth-factor-releasing hydrogels in an ex vivo chick femur defect model. Acta biomaterialia. 2014;10:4186-96.

[4] Smith EL, Kanczler JM, Gothard D, et al. Evaluation of skeletal tissue repair, part 2: enhancement of skeletal tissue repair through dual-growth-factor-releasing hydrogels within an ex vivo chick femur defect model. Acta biomaterialia. 2014;10:4197-205.

[5] Boden SD. Biology of lumbar spine fusion and use of bone graft substitutes: present, future, and next generation. Tissue engineering. 2000;6:383-99.

[6] Greenwald AS, Boden SD, Goldberg VM, et al. Bone-graft substitutes: facts, fictions, and applications. The Journal of bone and joint surgery American volume. 2001;83-A Suppl 2 Pt 2:98-103.

[7] Papadimitropoulos A, Scotti C, Bourgine P, et al. Engineered decellularized matrices to instruct bone regeneration processes. Bone. 2015;70c:66-72.

[8] Laurencin C, Khan Y, El-Amin SF. Bone graft substitutes. Expert review of medical devices. 2006;3:49-57.

[9] Polo-Corrales L, Latorre-Esteves M, Ramirez-Vick JE. Scaffold design for bone regeneration. Journal of nanoscience and nanotechnology. 2014;14:15-56.

[10] Hoganson DM, Meppelink AM, Hinkel CJ, et al. Differentiation of human bone marrow mesenchymal stem cells on decellularized extracellular matrix materials. Journal of biomedical materials research Part A. 2014;102:2875-83. 
[11] Trappmann B, Gautrot JE, Connelly JT, et al. Extracellular-matrix tethering regulates stem-cell fate. Nature materials. 2012;11:642-9.

[12] Tatullo M, Marrelli M, Falisi G, et al. Mechanical influence of tissue culture plates and extracellular matrix on mesenchymal stem cell behavior: A topical review. International journal of immunopathology and pharmacology. 2015.

[13] Jha AK, Jackson WM, Healy KE. Controlling osteogenic stem cell differentiation via soft bioinspired hydrogels. PloS one. 2014;9:e98640.

[14] Datta N, Holtorf HL, Sikavitsas VI, et al. Effect of bone extracellular matrix synthesized in vitro on the osteoblastic differentiation of marrow stromal cells. Biomaterials. 2005;26:971-7.

[15] Pham QP, Kasper FK, Scott Baggett L, et al. The influence of an in vitro generated bone-like extracellular matrix on osteoblastic gene expression of marrow stromal cells. Biomaterials. 2008;29:2729-39.

[16] Badylak SF. The extracellular matrix as a scaffold for tissue reconstruction. Seminars in cell \& developmental biology. 2002;13:377-83.

[17] Badylak SF, Freytes DO, Gilbert TW. Extracellular matrix as a biological scaffold material: Structure and function. Acta biomaterialia. 2009;5:1-13.

[18] Shekaran A, Garcia AJ. Extracellular matrix-mimetic adhesive biomaterials for bone repair. Journal of biomedical materials research Part A. 2011;96:261-72.

[19] Sawkins MJ, Bowen W, Dhadda P, et al. Hydrogels derived from demineralized and decellularized bone extracellular matrix. Acta biomaterialia. 2013;9:7865-73.

[20] Paduano F, Marrelli M, White LJ, et al. Odontogenic Differentiation of Human Dental Pulp Stem Cells on Hydrogel Scaffolds Derived from Decellularized Bone Extracellular Matrix and Collagen Type I. PloS one. 2016;11:e0148225.

[21] Gothard D, Smith EL, Kanczler JM, et al. In Vivo Assessment of Bone Regeneration in Alginate/Bone ECM Hydrogels with Incorporated Skeletal Stem Cells and Single Growth Factors. PloS one. 2015;10:e0145080.

[22] Gronthos S, Mankani M, Brahim J, et al. Postnatal human dental pulp stem cells (DPSCs) in vitro and in vivo. Proc Natl Acad Sci U S A. 2000;97:13625-30.

[23] Tatullo M, Falisi G, Amantea M, et al. DENTAL PULP STEM CELLS AND HUMAN PERIAPICAL CYST MESENCHYMAL STEM CELLS IN BONE TISSUE REGENERATION: COMPARISON OF BASAL AND OSTEOGENIC DIFFERENTIATED GENE EXPRESSION OF A NEWLY DISCOVERED 
MESENCHYMAL STEM CELL LINEAGE. Journal of biological regulators and homeostatic agents. 2015;29:713-8.

[24] Yang X, Walboomers XF, van den Beucken JJ, et al. Hard tissue formation of STRO1-selected rat dental pulp stem cells in vivo. Tissue Eng Part A. 2009;15:367-75.

[25] Graziano A, d'Aquino R, Cusella-De Angelis MG, et al. Scaffold's surface geometry significantly affects human stem cell bone tissue engineering. Journal of cellular physiology. 2008;214:166-72.

[26] Tatullo M, Marrelli M, Shakesheff KM, et al. Dental pulp stem cells: function, isolation and applications in regenerative medicine. Journal of tissue engineering and regenerative medicine. 2014.

[27] d'Aquino R, De Rosa A, Lanza V, et al. Human mandible bone defect repair by the grafting of dental pulp stem/progenitor cells and collagen sponge biocomplexes. Eur Cell Mater. 2009;18:75-83.

[28] Sano A, Hojo T, Maeda M, et al. Protein release from collagen matrices. Advanced drug delivery reviews. 1998;31:247-66.

[29] Londono R, Badylak SF. Biologic Scaffolds for Regenerative Medicine: Mechanisms of In vivo Remodeling. Annals of biomedical engineering. 2014.

[30] Wangensteen KJ, Kalliainen LK. Collagen tube conduits in peripheral nerve repair: a retrospective analysis. Hand (New York, NY). 2010;5:273-7.

[31] Qian J, Jiayuan W, Wenkai J, et al. Basic fibroblastic growth factor affects the osteogenic differentiation of dental pulp stem cells in a treatment-dependent manner. International endodontic journal. 2014.

[32] Shimabukuro Y, Ueda M, Ozasa M, et al. Fibroblast growth factor-2 regulates the cell function of human dental pulp cells. Journal of endodontics. 2009;35:1529-35.

[33] Tamama K, Kawasaki H, Wells A. Epidermal growth factor (EGF) treatment on multipotential stromal cells (MSCs). Possible enhancement of therapeutic potential of MSC. Journal of biomedicine \& biotechnology. 2010;2010:795385.

[34] Sapudom J, Rubner S, Martin S, et al. The phenotype of cancer cell invasion controlled by fibril diameter and pore size of 3D collagen networks. Biomaterials. 2015;52:367-75.

[35] Marrazzo P, Paduano F, Palmieri F, et al. Highly Efficient In Vitro Reparative Behaviour of Dental Pulp Stem Cells Cultured with Standardised Platelet Lysate Supplementation. Stem cells international. 2016;2016:7230987. 
[36] Marrelli M, Paduano F, Tatullo M. Human periapical cyst-mesenchymal stem cells differentiate into neuronal cells. Journal of dental research. 2015;94:843-52.

[37] Marrelli M, Paduano F, Tatullo M. Cells isolated from human periapical cysts express mesenchymal stem cell-like properties. International journal of biological sciences. 2013;9:1070-8.

[38] Paino F, La Noce M, Tirino V, et al. Histone deacetylase inhibition with valproic acid downregulates osteocalcin gene expression in human dental pulp stem cells and osteoblasts: evidence for HDAC2 involvement. Stem cells (Dayton, Ohio). 2014;32:27989.

[39] Paduano F, Marrelli M, Palmieri F, et al. CD146 Expression Influences Periapical Cyst Mesenchymal Stem Cell Properties. Stem cell reviews. 2016.

[40] Finkemeier CG. Bone-grafting and bone-graft substitutes. The Journal of bone and joint surgery American volume. 2002;84-a:454-64.

[41] Mangano C, Paino F, d'Aquino R, et al. Human dental pulp stem cells hook into biocoral scaffold forming an engineered biocomplex. PloS one. 2011;6:e18721.

[42] Penolazzi L, Mazzitelli S, Vecchiatini R, et al. Human mesenchymal stem cells seeded on extracellular matrix-scaffold: viability and osteogenic potential. Journal of cellular physiology. 2012;227:857-66.

[43] d'Aquino R, Graziano A, Sampaolesi M, et al. Human postnatal dental pulp cells codifferentiate into osteoblasts and endotheliocytes: a pivotal synergy leading to adult bone tissue formation. Cell Death Differ. 2007;14:1162-71.

[44] Laino G, d'Aquino R, Graziano A, et al. A new population of human adult dental pulp stem cells: a useful source of living autologous fibrous bone tissue (LAB). Journal of bone and mineral research : the official journal of the American Society for Bone and Mineral Research. 2005;20:1394-402.

[45] Davies OG, Cooper PR, Shelton RM, et al. A comparison of the in vitro mineralisation and dentinogenic potential of mesenchymal stem cells derived from adipose tissue, bone marrow and dental pulp. Journal of bone and mineral metabolism. 2014.

[46] Eleuterio E, Trubiani O, Sulpizio M, et al. Proteome of human stem cells from periodontal ligament and dental pulp. PloS one. 2013;8:e71101.

[47] Riccio M, Resca E, Maraldi T, et al. Human dental pulp stem cells produce mineralized matrix in 2D and 3D cultures. European journal of histochemistry : EJH. 2010;54:e46. 
[48] Mori G, Brunetti G, Oranger A, et al. Dental pulp stem cells: osteogenic differentiation and gene expression. Annals of the New York Academy of Sciences. 2011;1237:47-52.

[49] Otaki S, Ueshima S, Shiraishi K, et al. Mesenchymal progenitor cells in adult human dental pulp and their ability to form bone when transplanted into immunocompromised mice. Cell biology international. 2007;31:1191-7.

[50] Yang X, van der Kraan PM, van den Dolder J, et al. STRO-1 selected rat dental pulp stem cells transfected with adenoviral-mediated human bone morphogenetic protein 2 gene show enhanced odontogenic differentiation. Tissue engineering. 2007;13:2803-12.

[51] Papaccio G, Graziano A, d'Aquino R, et al. Long-term cryopreservation of dental pulp stem cells (SBP-DPSCs) and their differentiated osteoblasts: a cell source for tissue repair. Journal of cellular physiology. 2006;208:319-25.

[52] Bouet G, Bouleftour W, Juignet L, et al. The impairment of osteogenesis in bone sialoprotein (BSP) knockout calvaria cell cultures is cell density dependent. PloS one. 2015; 10:e0117402.

[53] Shakibaei M, Shayan P, Busch F, et al. Resveratrol mediated modulation of Sirt1/Runx2 promotes osteogenic differentiation of mesenchymal stem cells: potential role of Runx2 deacetylation. PloS one. 2012;7:e35712.

[54] Ichijima T, Matsuzaka K, Tonogi M, et al. Osteogenic differences in cultured rat periosteal cells under hypoxic and normal conditions. Experimental and therapeutic medicine. 2012;3:165-70.

[55] Joung YH, Lim EJ, Darvin P, et al. MSM enhances GH signaling via the Jak2/STAT5b pathway in osteoblast-like cells and osteoblast differentiation through the activation of STAT5b in MSCs. PloS one. 2012;7:e47477.

[56] Tu X, Rhee Y, Condon KW, et al. Sost downregulation and local Wnt signaling are required for the osteogenic response to mechanical loading. Bone. 2012;50:209-17. 


\section{Tables and Figures}

Table 1: Sequences of primers used for qRT-PCR.

\begin{tabular}{|c|c|c|}
\hline Gene & Sequence (5'-3') & NCBI Accession Number \\
\hline \multirow[t]{2}{*}{$R U N X-2$} & Forward: ATGTGTGTTTGTTTCAGCAGCA & NM_001024630.3 \\
\hline & Reverse: TCCCTAAAGTCACTCGGTATGTGTA & \\
\hline \multirow[t]{2}{*}{ BSP } & Forward: GCAGTAGTGACTCATCCGAAGAA & NM_004967.3 \\
\hline & Reverse: GCCTCAGAGTCTTCATCTTCATTC & \\
\hline \multirow[t]{2}{*}{ OPN } & Forward: CAGTTGTCCCCACAGTAGACAC & NM_001040058.1 \\
\hline & Reverse: GTGATGTCCTCGTCTGTAGCATC & \\
\hline \multirow[t]{2}{*}{ OCN } & Forward: TGAGAGCCCTCACACTCCTC & NM_199173.4 \\
\hline & Reverse: ACCTTTGCTGGACTCTGCAC & \\
\hline \multirow[t]{2}{*}{ HPRT } & Forward: TGACACTGGCAAAACAATGCA & NM_000194.2 \\
\hline & Reverse: GGTCCTTTTCACCAGCAAGCT & \\
\hline
\end{tabular}

$\boldsymbol{R} \boldsymbol{U N X}-2$, runt-related transcription factor 2; BSP, bone sialoprotein; $\boldsymbol{O P N}$, osteopontin; $\boldsymbol{O C N}$, osteocalcin; HPRT, hypoxanthine phosphoribosyltransferase. 


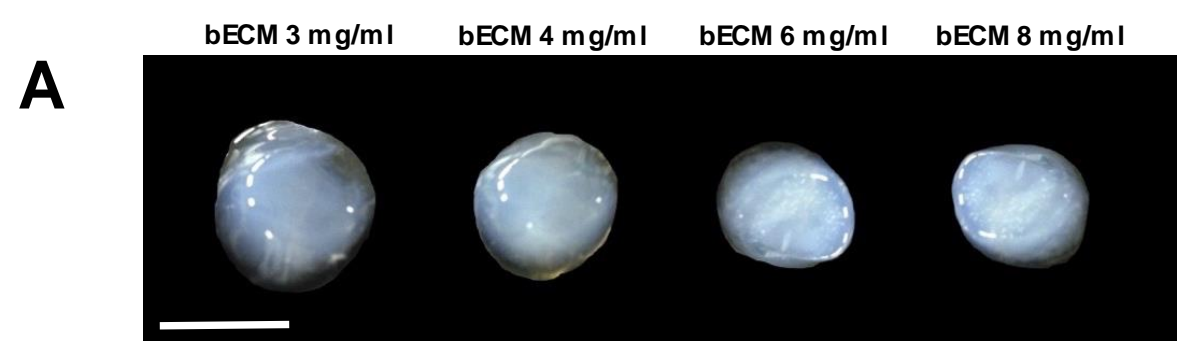

B

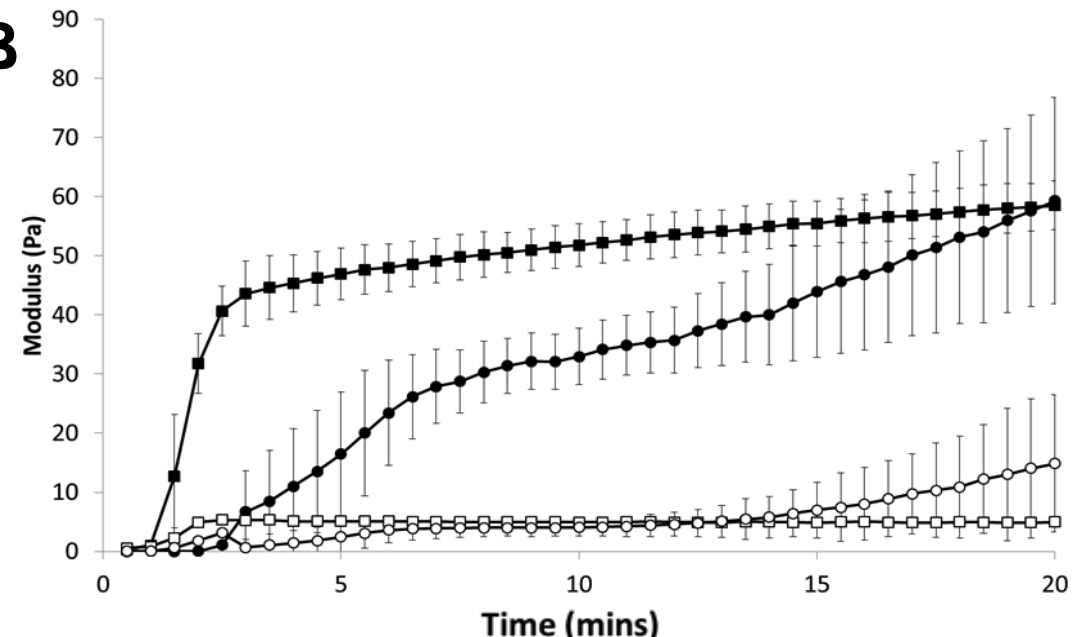

-Collagen $4 \mathrm{mg} / \mathrm{ml} \mathrm{G}^{\prime} \quad \rightarrow$ ECM 4 mg/ml G' $\rightarrow$-Collagen $4 \mathrm{mg} / \mathrm{ml} \mathrm{G"} \quad$-ECM 4 mg/ml G"

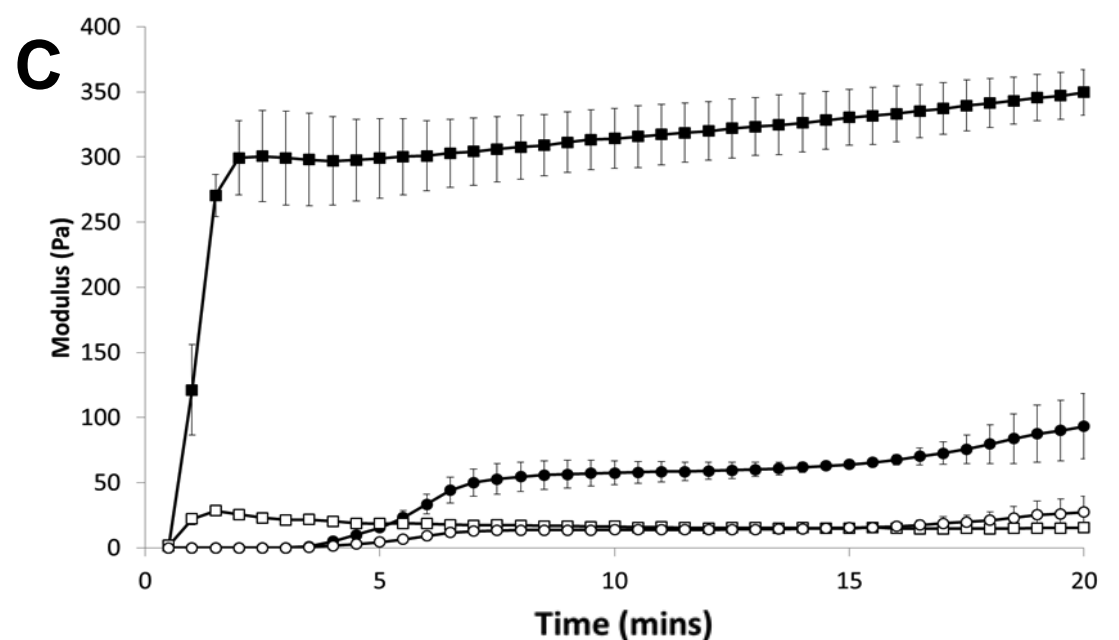

-Collagen $6 \mathrm{mg} / \mathrm{ml} \mathrm{G'} \rightarrow$ ECM 6 mg/ml G' $\multimap$-Collagen 6 mg/ml G" $\quad$-ECM 6 mg/ml G"

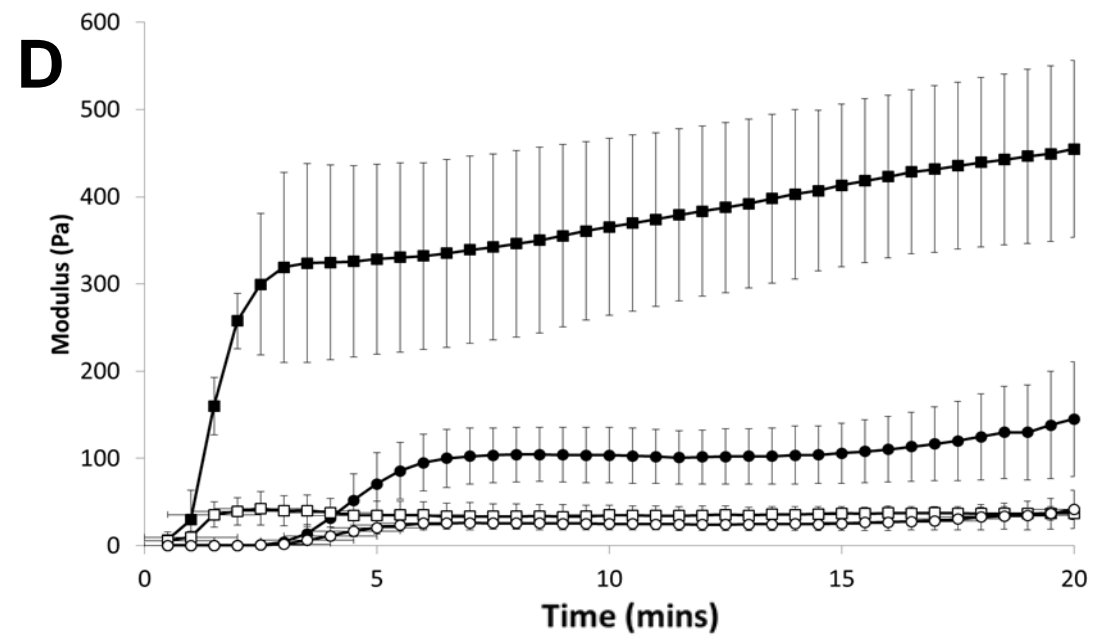

-Collagen $8 \mathrm{mg} / \mathrm{ml} \mathrm{G}^{\prime} \quad \rightarrow$ ECM 8 mg/ml G' $\quad$-Collagen $8 \mathrm{mg} / \mathrm{ml} \mathrm{G"} \quad$-ECM 8 mg/ml G" 


\section{Figure 1. Macroscopic appearance and rheology of bECM hydrogels.}

A) Macroscopic view of bECM hydrogels at $3 \mathrm{mg} / \mathrm{mL}, 4 \mathrm{mg} / \mathrm{mL}, 6 \mathrm{mg} / \mathrm{mL}$ and $8 \mathrm{mg} / \mathrm{mL}$, scale bar $=1 \mathrm{~cm}$. Rheological characterization of bECM and collagen type I hydrogels at concentrations of B: $4 \mathrm{mg} / \mathrm{mL}, \mathbf{C}: 6 \mathrm{mg} / \mathrm{mL}$ and $\mathbf{D}: 8 \mathrm{mg} / \mathrm{mL}$. Gelation kinetics were determined by monitoring changes in the storage modulus $\left(\mathrm{G}^{\prime}\right)$ and loss modulus $\left(\mathrm{G}^{\prime \prime}\right)$ after inducing gelation. Data represent mean \pm standard deviation for $\mathrm{n}=3$. 

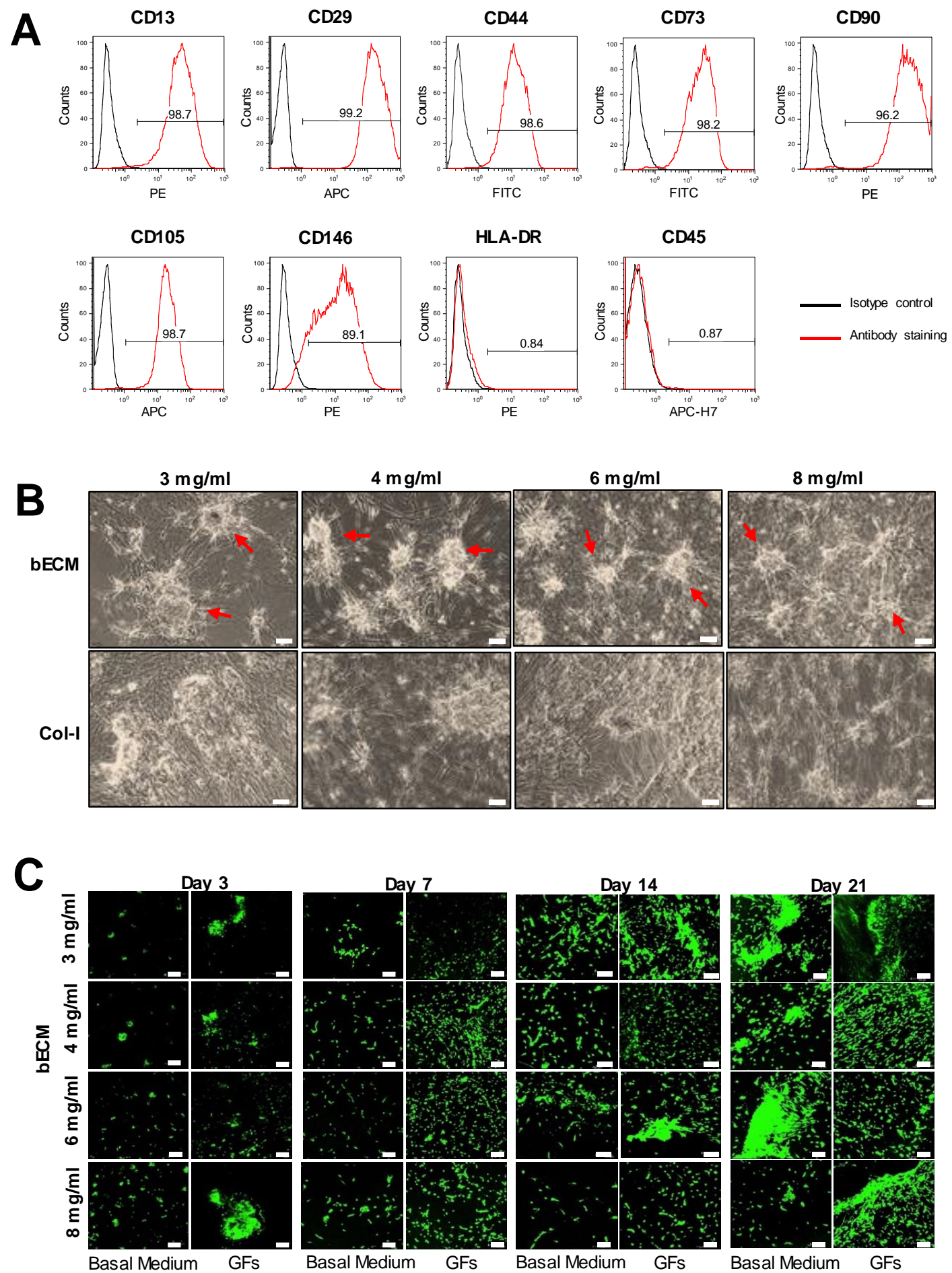

D

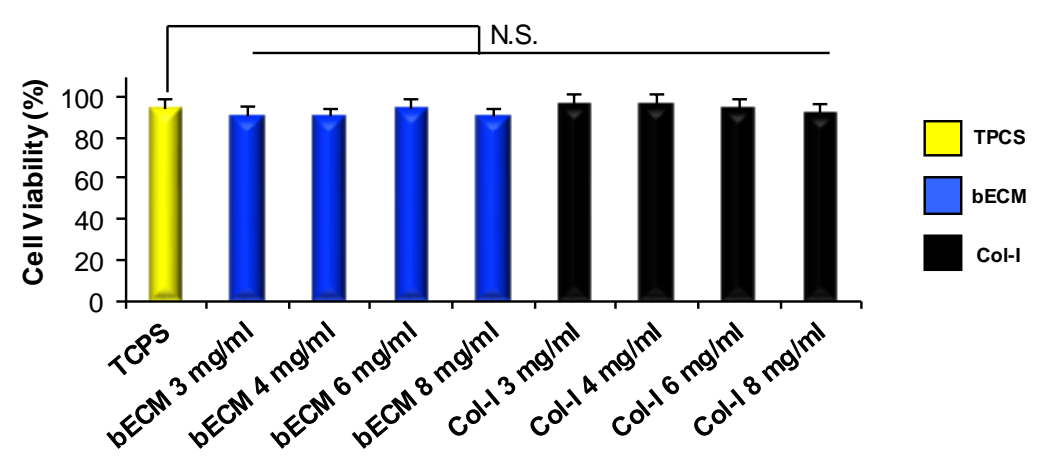




\section{Figure 2. bECM supports cell attachment and growth of DPSCs.}

A) Flow cytometry analysis of MSC surface markers CD13, CD29, CD44, CD73, CD90, CD105, CD146, HLA-DR and CD45 for DPSCs cultured on bECM. The percentage of positive cells for each marker is shown, and the isotype control is indicated. The logarithm of the $\mathrm{X}$-axis represents the intensity of the fluorescent signal, and the number of cells is given on the Y-axis. B) Representative distribution of DPSCs cultured on bECM $(3,4,6$, $8 \mathrm{mg} / \mathrm{mL})$, Col-I hydrogels $(3,4,6,8 \mathrm{mg} / \mathrm{mL})$. Cultures were photographed after 3 weeks, scale bar $=100 \mu \mathrm{m}$. Red arrows indicate cell aggregates. C) Live/dead analysis of cells/scaffold constructs 3, 7, 14 and 21 days after cell seeding in basal medium or in medium containing growth factors (FGFb + EGF) by confocal laser scanning microscopy. D) Percentages of cell viability at day 7 of DPSCs cultured on bECM $(3,4,6,8 \mathrm{mg} / \mathrm{mL})$ hydrogels with respect to Col-I $(3,4,6,8 \mathrm{mg} / \mathrm{mL})$ hydrogels or TCPS measured by the PI method using cell counter. N.S. Not statistically significant. 

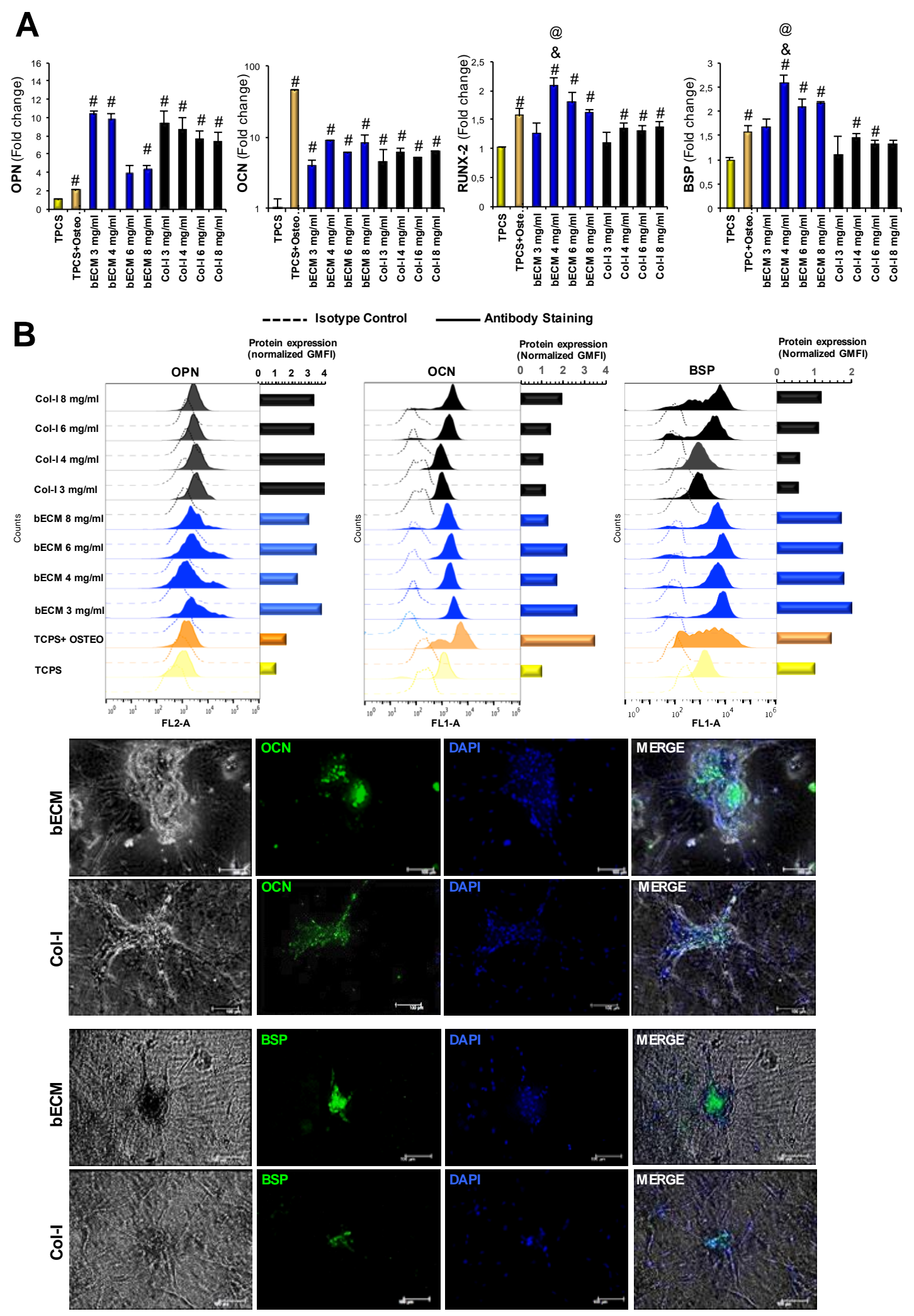

Figure 3. bECM hydrogels upregulate osteogenic markers of DPSCs in basal culture conditions. 
A) Relative mRNA expression levels of $R U N X-2, B S P, O P N$ and $O C N$ of DPSCs at 3 weeks measured by qRT-PCR. DPSCs were cultured on bECM and Col-I hydrogels at different concentrations $(3,4,6,8 \mathrm{mg} / \mathrm{mL})$ in the basal medium compared to those seeded on TCPS and cultured in osteogenic medium. Results are represented as fold increase compared to the level expressed in DPSCs seeded on TCPS cultured in the basal medium. ${ }^{\#} P<0.05$ when compared to TCPS; ${ }^{\&} P<0.05$ when compared to $4 \mathrm{mg} / \mathrm{mL}$ Col-I hydrogel; ${ }^{\circledR} P<0.05$ when compared to TCPS cultured in osteogenic medium. B) Flow cytometry analysis at 3 weeks of OPN, OCN and BSP proteins expression in DPSC populations seeded on bECM $(3,4,6,8 \mathrm{mg} / \mathrm{mL})$, Col-I $(3,4,6,8 \mathrm{mg} / \mathrm{mL})$ hydrogels or TCPS cultured in basal medium compared to those seeded on TCPS cultured in osteogenic medium. Cells were labelled with fluorescence-conjugated antibodies against OPN, OCN (solid histograms) or with isotype controls (dotted histograms). For BSP, cells were labelled with an antibody against BSP (solid lines) or with isotype control (dotted lines), followed by detection with Alexa 488-conjugated secondary antibody. Histograms show the geometric mean fluorescence intensity (GMFI) expressed as fold increase with respect to TCPS. Flow cytometry data were analysed using FlowJo vX0.7 software. C) Representative immunofluorescence analysis of DPSCs cultured on $4 \mathrm{mg} / \mathrm{mL}$ bECM and $4 \mathrm{mg} / \mathrm{mL}$ Col-I hydrogels stained with OCN and BSP after 3 weeks in basal medium. OCN, osteocalcin; BSP, bone sialoprotein. The cell nuclei were stained with DAPI (blue); scale bar $=100 \mu \mathrm{m}$. 
A

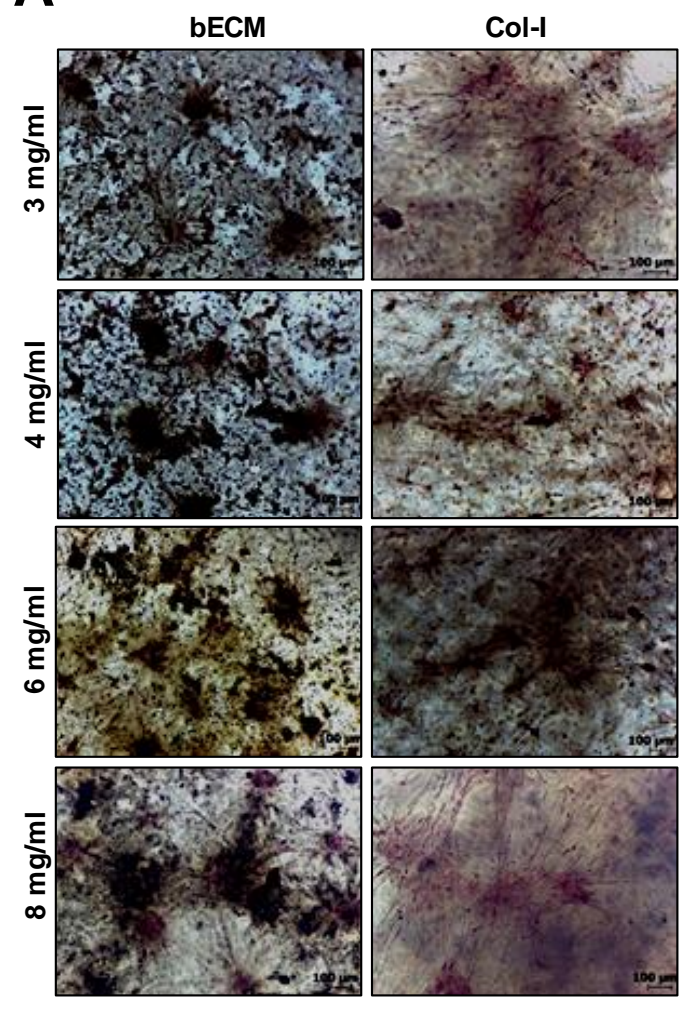

Von Kossa
B

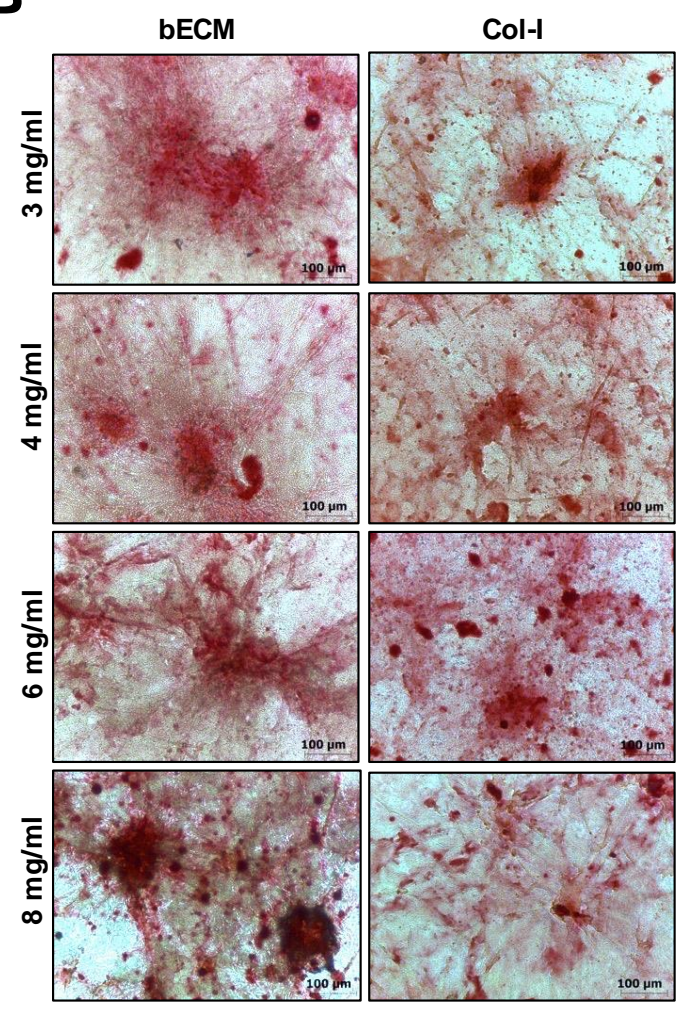

Alizarin Red

Figure 4. Mineral deposition of DPSCs/bECM and DPSCs/Col-I hydrogel constructs.

Von kossa (A) and Alizarin Red staining (B) of DPSCs cultured on bECM (3, 4, 6, 8 $\mathrm{mg} / \mathrm{mL})$ and Col-I $(3,4,6,8 \mathrm{mg} / \mathrm{mL})$ hydrogels in basal medium after 3 weeks; scale bar $=100 \mu \mathrm{m}$. 
A

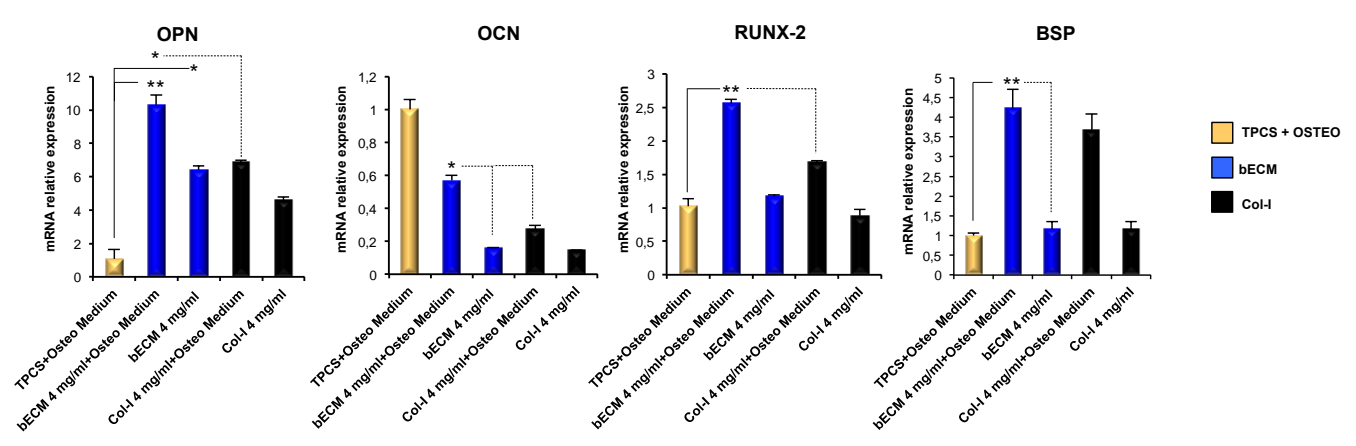

B

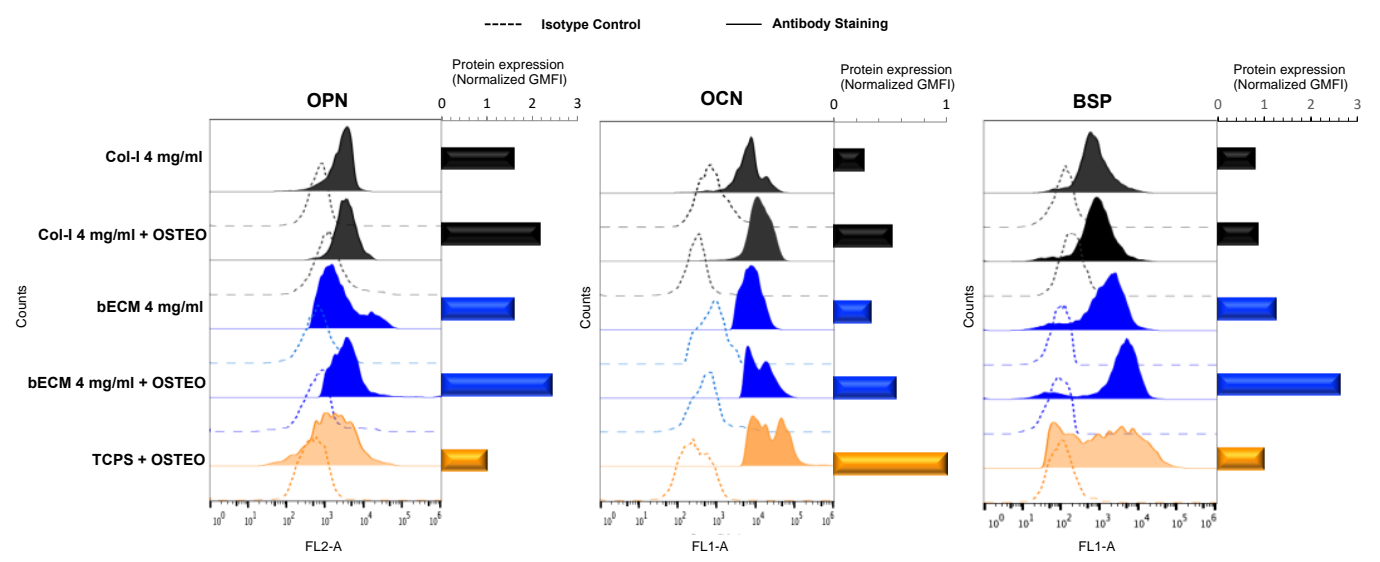

Figure 5. Osteogenic supplements enhance osteogenic differentiation of DPSCs/bECM hydrogel constructs.

A) Relative mRNA expression levels of $R U N X-2, B S P, O P N$ and $O C N$ of DPSCs at 3 weeks measured by qRT-PCR. DPSCs were cultured on $4 \mathrm{mg} / \mathrm{mL}$ bECM, 4 mg/mL ColI and TCPS cultured in osteogenic medium. Data are represented as fold increase compared to the level expressed in DPSCs seeded on TCPS cultured in osteogenic medium. ${ }^{\circledR} P<0.05$ when compared to TCPS cultured in osteogenic medium; ${ }^{\$} P<0.05$ when compared to bECM $4 \mathrm{mg} / \mathrm{mL}$ cultured in basal medium and ${ }^{\&} P<0.05$ when compared to Col-I $4 \mathrm{mg} / \mathrm{mL}$ cultured in osteogenic medium. B) Flow cytometry analysis at 3 weeks of OPN, OCN and BSP expression in DPSC populations seeded on $4 \mathrm{mg} / \mathrm{mL}$ bECM, $4 \mathrm{mg} / \mathrm{mL}$ Col-I and TCPS cultured in osteogenic medium. Cells were labelled with antibodies against OPN, OCN and BSP (solid histograms) or with isotype controls 
(dotted histograms). Histograms show the geometric mean fluorescence intensity (GMFI) expressed as fold increase with respect to TCPS cultured in osteogenic medium. 
A

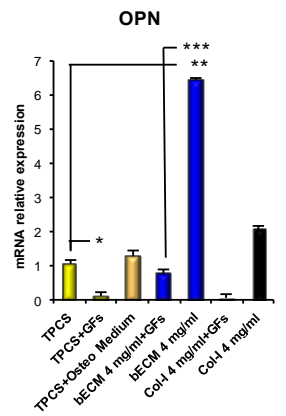

OCN
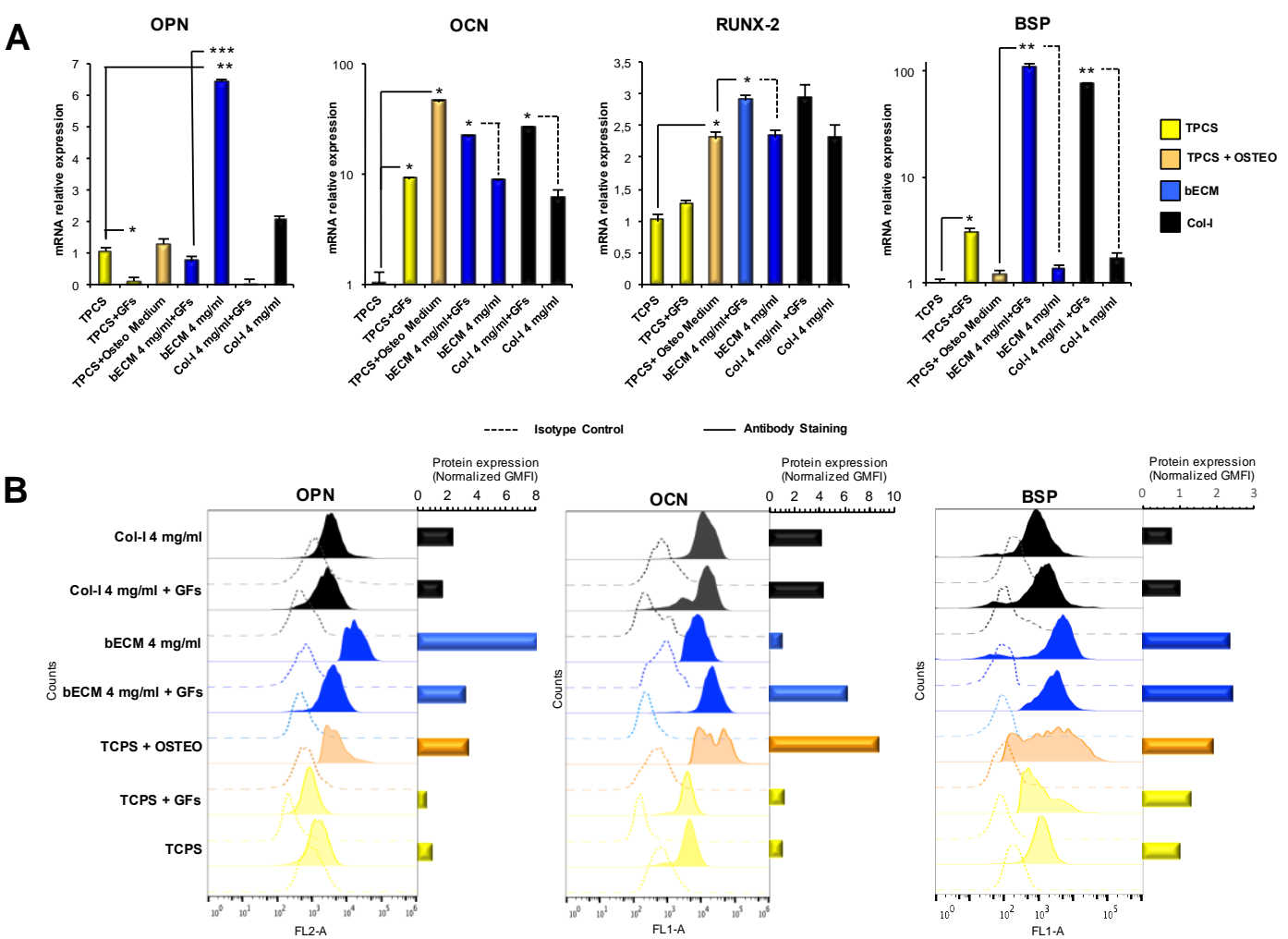

Figure 6. GFs affect the osteogenic differentiation of DPSCs in combination with bECM hydrogels.

A) Relative mRNA expression levels of $R U N X-2, B S P, O P N$ and $O C N$ of DPSCs at 3 weeks measured by qRT-PCR. Cells were cultured on $4 \mathrm{mg} / \mathrm{mL}$ bECM or Col-I hydrogel scaffolds in medium supplemented with growth factors or basal medium. Data are shown as the fold increase compared to the level expressed in DPSCs cultured on TCPS in basal medium. ${ }^{\#} P<0.05$ when compared to TCPS cultured in medium containing GFs; ${ }^{\$} P<$ 0.05 when compared to the respective hydrogels in basal medium; ${ }^{\&} P<0.05$ when compared to TCPS cultured in osteogenic medium. B) Flow cytometry analysis at 3 weeks of OPN, OCN and BSP expression in DPSCs seeded on $4 \mathrm{mg} / \mathrm{mL}$ bECM or 4 $\mathrm{mg} / \mathrm{mL}$ Col-I hydrogels cultured in medium containing GFs (growth factors; FGFb + EGF) compared to those seeded on TCPS cultured in basal or osteogenic medium. Cells were labelled with fluorescence-conjugated antibodies against OPN, OCN (solid 
histograms) or with isotype controls (dotted histograms). For BSP, cells were labelled with an antibody against BSP (solid lines) or with isotype control (dotted lines), followed by detection with Alexa 488-conjugated secondary antibody. Histograms show the geometric mean fluorescence intensity (GMFI) expressed as fold increase with respect to TCPS cultured in the basal medium. Flow cytometry data were analysed using FlowJo vX0.7 software. 